This is a author version of

S. Raach, D. Schlipf, F. Sandner, D. Matha and P. W. Cheng,

"Nonlinear Model Predictive Control of Floating Wind Turbines with Individual Pitch Control,"

published in Proceedings of the American Control Conference, Portland, USA, 2014.

\title{
Nonlinear Model Predictive Control of Floating Wind Turbines with Individual Pitch Control
}

\author{
Steffen Raach ${ }^{1}$, David Schlipf ${ }^{1}$, Frank Sandner ${ }^{1}$, Denis Matha ${ }^{1}$ and Po Wen Cheng ${ }^{1}$
}

\begin{abstract}
In this work a nonlinear model predictive controller with individual pitch control for a floating offshore wind turbine is presented. An aerodynamic model of the collective pitch control approach is extended by describing pitching and yawing moments based on rotor disk theory. This extension is implemented in a reduced nonlinear model of the floating wind turbine including disturbance preview of wind speed, linear vertical and horizontal wind shear, and wave height to compute optimal input trajectories for the individual pitch control inputs and the generator torque. An extended cost functional for individual pitch control is proposed based on the collective pitch control approach. The controller is evaluated in aero-servohydro-elastic simulations of a $5 \mathrm{MW}$ reference wind turbine disturbed by a three-dimensional stochastic turbulent wind field. Results show a significant blade fatigue load reduction compared to a baseline controller through minimizing yawing and pitching moments on the rotor hub while maintaining the advantages of the model predictive control approach with collective pitch control.
\end{abstract}

\section{INTRODUCTION}

Offshore wind energy can play a decisive role in global alternative energy production. But current fixed-bottom support structures have limitations in terms of maximum depth and require flatness of the seabed. Therefore, a lot of research has been conducted in the area of floating offshore wind turbines (FOWTs) in the last years. The idea behind this concept is to mount the wind turbine on a floating platform which is moored to the seabed, see [1], [2], [3], [4]. This concept enables offshore wind energy to exploit the vast deep water regions common in many areas of the world.

In various publications on modeling floating wind turbines control issues are mentioned and the interaction between the pitch controller and the platform motion is pointed out to be not well or even negatively damped, see [1], [2], [5], [6], [7]. These stability problems are important and occur when a platform with very low natural frequency of the rigid body modes is combined with a traditional state of the art pitch controller. Thus, for FOWTs the controller has to be adjusted as mentioned by [5] and [8] to guarantee stability of the system. Further, new concepts in platform design can improve stability, see [9], [10]. Fischer [11] gave an analysis on the coupling between platform motion and pitch controller and mentioned a complex pair of non-minimum phase zeros near the natural frequency of the platform pitch eigenmode. A straight forward approach to deal with this is to lower the closed-loop bandwidth of the pitch controller under the

\footnotetext{
${ }^{1}$ Stuttgart Chair of Wind Energy (SWE), University of Stuttgart, Stuttgart, Germany Steffen.Raach/David.Schlipf/ Frank. Sandner/Denis. Matha/PoWen. Cheng at ifb.uni-stuttgart.de
}

platform pitch frequency as done in [2] and [5] which results in an increased rotational speed variation which is up to $30 \%$ higher than nominal speed. Another approach is to use gain scheduling as mentioned in [8]. A big disadvantage in gain scheduling is the fact that stability is not guaranteed, see[8]. [12] proposes an approach in which a basic pitch controller is augmented by an increment pitch angle controller. The presented approaches are mainly frequency-based controller design methods. There are several approaches in model based control for floating wind turbines like the LQ approach in [8], an $H \infty$ approach in [13], the variable power collective pitch approach in [14]. [15] uses an individual pitch control (IPC) and an approach in which a periodic state space controller is used to control the turbine. A model predictive approach with IPC is used in [16] where also uncertain wind measurements and lead-lag errors were considered. Nonlinear model predictive control (NMPC) with collective pitch control (CPC) has shown good results for onshore wind turbines by [17], [18], and [19] and recently this approach was adapted to FOWT and successfully used in [20]. In summary, model based approaches presented in literature show a promising way to solve the problems of floating wind turbines.

NMPC seems to be a valuable technique for FOWT control because of its possibility to include constraints and its concept to take disturbance predictions into account. Furthermore, it offers the possibility for the controller to use its inputs in such a way that the control goal is reached best. But translating the desired control requirements to a mathematical representation for the cost functional is the crucial part of designing the controller.

The motivation for an IPC control is to reduce blade loads by minimizing yawing and pitching moments of the rotor. As a side effect an improvement in control performance and reduction of the total load of the FOWT is expected. Hence, an extension is purposed, a NMPC IPC controller to reduce blade loads and to open additional DOFs for the controller.

This paper is organized as follows. Section II describes the simulation environment and focuses on the derivation of a reduced model for a FOWT. In Section III the NMPC controller is introduced and a cost functional for a FOWT with IPC is presented. Simulation results are presented in Section IV, and a conclusion and perspectives on future work are given in Section V. Altogehter, this paper focuses on the control problem of a FOWT and the performance an optimal controller can provide. Therefore, this contribution can be used as a benchmark for prospective controller. 


\section{MODELING}

In this work a $5 \mathrm{MW}$ turbine on a spar-buoy is considered, see [2]. The FOWT is implemented in the simulation tool FAST [21] described in Section II-A and in a reduced model presented in Section II-B. In the first implementation the FOWT is disturbed by a 3D stochastic turbulent wind field and irregular waves while the reduced model with reduced disturbance inputs is used in the controller to calculate optimal input trajectories.

\section{A. Full Model for Simulations}

The coupled FAST model for FOWTs consists of a flexible multibody system with a total of 22 DOFs. A servoelastic structural model is coupled with models computing the external aerodynamic, hydrodynamic, and mooring line forces. The hydrodynamic model is based on linear potential flow theory with the viscous damping term of Morison's equation. Using BEM (Blade Element Momentum) theory aerodynamic forces from the interaction of the rotor with an incoming turbulent wind field (discretized in a cartesian rectangular grid) are computed. The mooring forces are calculated by solving iteratively a quasi-static equation for a slack line. The described model has proven reliable accuracy (see [2]) and is therefore applicable to validate the NMPC based on the reduced internal model.

\section{B. Reduced Internal Model for Controller Design}

The presented reduced model for IPC is an extension to the reduced model of [20] which is a simplification of [3]. The reduced disturbances are the wind speed $v_{0}$, the linear vertical and horizontal wind shear $\delta_{V}$ and $\delta_{H}$, and the wave height $\eta$.

1) Servo-Elastics: The FOWT is modeled as coupled system of $p=4$ rigid bodies, platform, tower, nacelle and rotor with a total number of $f=4$ DOFs, which are the platform position $x_{P}$, the relative tower-top position $x_{T}$ in direction of the wind, the platform pitch angle $\theta_{P}$, and the azimuth angle of the rotor $\psi$. These general coordinates are comprised in the vector

$$
\boldsymbol{q}=\left[\begin{array}{llll}
x_{P} & \theta_{P} & x_{T} & \psi
\end{array}\right]^{T} .
$$

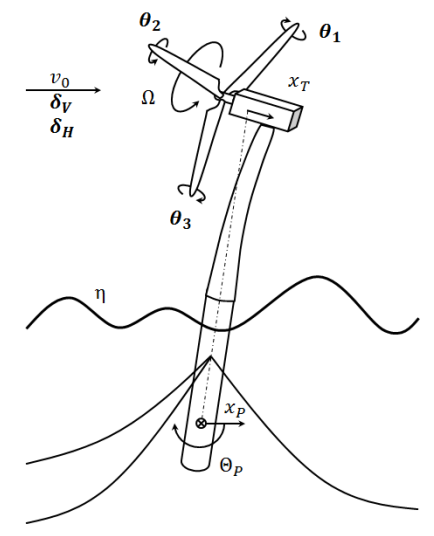

Fig. 1. Considered DOFs and disturbances of the reduced internal model.
Fig. 1 shows the reduced model with its DOFs, where $\Omega=\dot{\psi}$ is the rotor speed. Following the formalism of [22] the equation of motion can be obtained by applying Newton's Second Law as well as Euler's law to each body in all spacial directions. According to [22] this results in a $(2 \cdot 3 p)$ dimensional system of equations,

$$
\boldsymbol{M}(\boldsymbol{q}) \cdot \boldsymbol{J}(\boldsymbol{q}) \cdot \ddot{\boldsymbol{q}}+\boldsymbol{k}(\boldsymbol{q}, \dot{\boldsymbol{q}})=\boldsymbol{p}(\boldsymbol{q}, \dot{\boldsymbol{q}})+\boldsymbol{Q}(\boldsymbol{q}, \dot{\boldsymbol{q}}) \cdot \boldsymbol{g}(\boldsymbol{q}, \dot{\boldsymbol{q}}),
$$

where in $\boldsymbol{M}$ the body masses and the moment of inertia of the bodies are arranged. $\boldsymbol{J}$ is the global Jacobian matrix, $\boldsymbol{k}$ contains the coriolis-, centrifugal and gyroscopic part of the Newton-Euler equation, $\boldsymbol{p}$ contains all external applied forces and moments like the generator torque $M_{g}$, and in $\boldsymbol{Q} \cdot \boldsymbol{g}$ all reaction forces and coupling forces between bodies are summarized. The reaction forces can be eliminated by multiplying (2) with the transposed global Jacobian matrix $\boldsymbol{J}^{T}$ from the left since orthogonality yields $\boldsymbol{J}^{T} \cdot Q=0$. It remains the $(f \times f)$ mass matrix $\overline{\boldsymbol{M}}=\overline{\boldsymbol{M}}^{T}>0$ being positive definite after the transformation and the vectors $\boldsymbol{k}$ and $\boldsymbol{p}$ result in vector $\overline{\boldsymbol{k}}$ and $\overline{\boldsymbol{p}}$. Thus, a set of differential equations

$$
\overline{\boldsymbol{M}}(\boldsymbol{q}) \cdot \ddot{\boldsymbol{q}}+\overline{\boldsymbol{k}}(\boldsymbol{q}, \dot{\boldsymbol{q}})=\overline{\boldsymbol{p}}(\boldsymbol{q}, \dot{\boldsymbol{q}}),
$$

the equation of motion of the FOWT are gained. We obtain the state space representation of the FOWT by inverting the mass matrix $\overline{\boldsymbol{M}}$ :

$$
\dot{\boldsymbol{x}}=\left[\begin{array}{c}
\dot{\boldsymbol{q}} \\
\ddot{\boldsymbol{q}}
\end{array}\right]=\left[\begin{array}{c}
\dot{\boldsymbol{q}} \\
\overline{\boldsymbol{M}}^{-1}(\overline{\boldsymbol{p}}(\boldsymbol{q}, \dot{\boldsymbol{q}})-\overline{\boldsymbol{k}}(\boldsymbol{q}, \dot{\boldsymbol{q}}))
\end{array}\right] .
$$

Every blade can be controlled by an individual blade pitch angle $\theta_{i}$, see Fig. 1. Rotating states can be transformed in a nonrotating representation using a transformation (Coleman transformation) [23]. Therefore, horizontal and vertical blade pitch angle, $\theta_{H}$ and $\theta_{V}$, and a static blade pitch angle with respect to the azimuth $\psi$ are defined. Thus, the blade pitch angles are

$$
\left[\begin{array}{l}
\theta_{1} \\
\theta_{2} \\
\theta_{3}
\end{array}\right]=\left[\begin{array}{ccc}
1 & \sin \psi & \cos \psi \\
1 & \sin \left(\psi+\frac{2}{3} \pi\right) & \cos \left(\psi+\frac{2}{3} \pi\right) \\
1 & \sin \left(\psi+\frac{4}{3} \pi\right) & \cos \left(\psi+\frac{4}{3} \pi\right)
\end{array}\right]\left[\begin{array}{c}
\theta \\
\theta_{V} \\
\theta_{H}
\end{array}\right] .
$$

The idea behind this transformation is to obtain a fixed nonrotating coordinate system, in which control actions can be assigned directly to the different transformed states and the controller can be designed in a straight forward way. Thus, this transformation offers not only a great possibility for control purposes but also for modeling because of the advantage to neglect a model for individual blades and therefore to use rotor disk theory. Fig. 2 shows the structural overview of the reduced model for IPC with its disturbances, its inputs, and the interaction between the different subsystems.

2) Reduced Mooring Line Model: To obtain the interaction between the mooring lines a quasi-static equation for a slack line is solved offline as a function of horizontal and vertical displacements. The result is fitted with a polynomial approach to gain a continuously differentiable function, which can be evaluated during runtime.

3) Reduced Hydrodynamic Model: A reduced model is used based on Morison's equation with simplifications to use the current wave height $\eta$ as disturbance input. This 


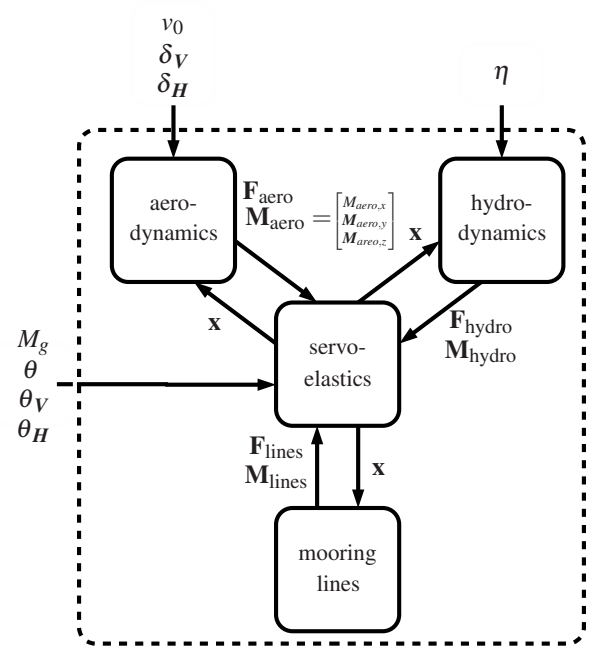

Fig. 2. The structural interaction between the subsystems of the reduced model and its control inputs and disturbances.

method of disturbance reduction has been implemented and evaluated in detail in [3].

4) Aerodynamics: Aerodynamics are based on rotor disk theory. The power and thrust coefficients are gained in a preprocessing step and fitted to a polynomial. With the rotor effective wind speed $v_{0}$ the aerodynamic thrust and torque can be calculated, see [24].

As an extension for IPC a nonlinear static model is introduced. It describes the pitching moment $M_{\text {Aero,y }}$ and yawing moment $M_{\text {Aero }, z}$ of the rotor to realize an individual blade pitching in nonrotating coordinates. The benefit of this approach is to gain a direct relationship between disturbances rotor effective wind speed $v_{0}$, vertical and horizontal wind shear, $\delta_{V}$ and $\delta_{H}$, and the blade control inputs $\theta, \theta_{V}$, and $\theta_{H}$. In relationship to the rotor disk theory the moments can be written as

$$
\begin{aligned}
& M_{\text {Aero, } y}=\frac{\rho \pi R^{2}}{2}\left(c_{S 1}\left(\delta_{V}-K_{\theta_{V H}} \theta_{V}\right)+c_{S 2} \delta_{H}\right) v_{0}^{2} \\
& M_{\text {Aero, } z}=\frac{\rho \pi R^{2}}{2}\left(c_{S 1}\left(\delta_{H}-K_{\theta_{V H}} \theta_{H}\right)+c_{S 2} \delta_{V}\right) v_{0}^{2},
\end{aligned}
$$

where $\rho$ is the air density and $R$ the rotor radius. $K_{\theta_{V H}}$ depends on the collective pitch angle $\theta$ and describes the nonlinearity and changed sensitivity at higher wind speeds. The state dependent influence parameter $c_{S 1}(\lambda, \theta, \gamma)$ and $c_{S 2}(\lambda, \theta, \gamma)$ describe the major and the minor coupling, respectively. The tip speed ratio is described by $\lambda$ and $\gamma$ is the misalignment angle of the wind direction with respect to the rotor. To cope with oblique inflow the rotor effective wind speed is corrected with the cosine of the misalignment angle, $v_{0, c}=v_{0} \cos \gamma$.

Fig. 3 depicts the major influence coefficient $c_{S 1}$ identified with the BEM module AeroDyn [25] for $\lambda \in[1,18]$ and $\theta \in[0,30] \mathrm{deg}$. The influence coefficients are also fitted to a polynomial to guarantee a fast evaluation during runtime. For validating the obtained model for pitching and yawing moment Fig. 4 shows a PSD evaluation of a $600 \mathrm{~s}$ simulation with FAST with a stochastic turbulent 3D wind field and the reduced model. The reduced disturbances, $v_{0}$ and the

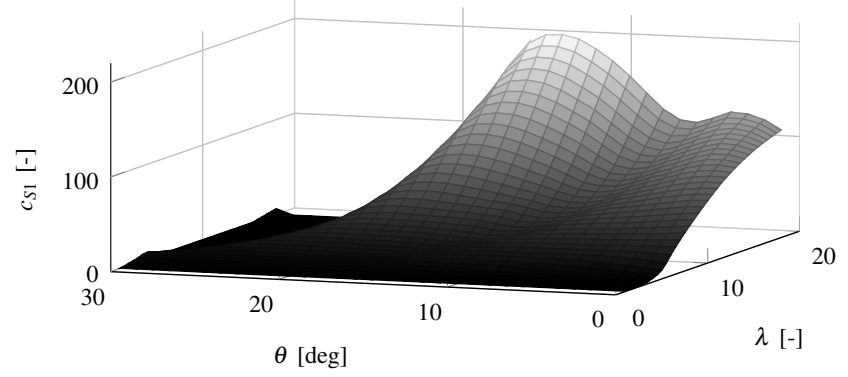

Fig. 3. Main influence coefficient $c_{S 1}(\lambda, \theta)$ describing the major coupling between shear and moment for $\lambda \in[1,18]$ and $\theta \in[0,30] \mathrm{deg}$.
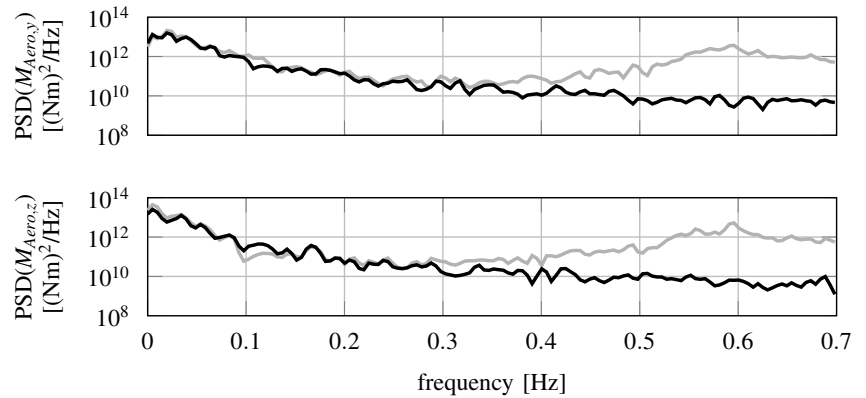

Fig. 4. PSD comparison between the reduced model (black) with the reduced wind disturbances and the complex model of FAST (light gray) with the 3D turbulent wind field.

vertical and horizontal wind shear $\delta_{V}$ and $\delta_{H}$, are extracted from the turbulent wind field using a weighted function. Low frequencies up to $0.2 \mathrm{~Hz}(1 \mathrm{P})$ are covered well representing the asymetric loading of the rotor being the main focus of the modeling. The peak at $0.6 \mathrm{~Hz}(3 \mathrm{P})$ can only reduced with a higher order controller, see [26].

\section{CONTROLLER DESIGN}

In this section the NMPC controller is derived using the wind speed, vertical and horizontal wind shear and wave height preview information.

\section{A. Problem Setup}

A NMPC controller predicts the future behavior using a internal model, the current measurements and the predictions of the disturbances to obtain an optimal input trajectory. Thus, the core of a NMPC controller is a repetitive solving of an optimal control problem and applying a defined time span of the calculated optimal input trajectory. Part of the solution is applied as input and new measurements are made to use as initial conditions for the optimal control problem. Input and stage constraints can be taken into account as well as multi-input and multi-output (MIMO). The inputs are the IPC blade inputs $\theta_{V}, \theta_{H}$, the blade pitch velocity $\dot{\theta}$ and the time derivative of the generator torque $\dot{M}_{g}$, allowing a limitation of the changing rate.

The considered optimal FOWT control problem with IPC can be described as follows. The objective is to find the optimal control trajectory $\boldsymbol{u}(\cdot)$ in the presence of the disturbance $\boldsymbol{d}(\cdot)$, minimizing the cost functional $J$, which is 
defined over the time horizon $T$ of the objective function $\Pi$ from the actual time $t_{0}$ to the final time $t_{0}+T$ with the reduced nonlinear model and the set of constraints $H$ :

$$
\begin{aligned}
& \min _{\boldsymbol{u}(\cdot)} J(\boldsymbol{x}, \boldsymbol{u}, \boldsymbol{d}) \\
\text { with: } & J(\boldsymbol{x}, \boldsymbol{u}, \boldsymbol{d})=\int_{t_{0}}^{t_{0}+T} \Pi(\boldsymbol{x}(\tau), \boldsymbol{u}(\tau), \boldsymbol{d}(\tau)) d \tau \\
\text { s.t. } \quad & \dot{\boldsymbol{x}}=f(\boldsymbol{x}, \boldsymbol{u}, \boldsymbol{d}) \\
& \boldsymbol{x}\left(t_{0}\right)=x_{0} \\
& H(\boldsymbol{x}(\tau), \boldsymbol{u}(\tau), \boldsymbol{d}(\tau)) \geq 0 \forall \tau \in\left[t_{0}, t_{0}+T\right] .
\end{aligned}
$$

Finding the right mathematical representation for the control goals and translating it to the cost functional and constraints is the crucial part in designing a NMPC controller. Generally, the goal of wind turbine control can be described as maximum possible power output with minimal structural loads in the feasible operation range. Here, above rated wind conditions are considered. In classic wind turbine control this is general done by limiting rotor speed and power above rated wind speed, see [23].

The objective function $\Pi$ is designed in a quadratic manner and the weights are independent of the system states and inputs. Thus, the objective function is

$$
\begin{aligned}
& \Pi(\boldsymbol{x}(t), \boldsymbol{u}(t), \boldsymbol{d}(t))=Q_{1} \\
& +Q_{2} \\
& +Q_{3} \\
& +Q_{4} \\
& +R_{1}\left(v_{0}(t)\right) \\
& +R_{2} \\
& +R_{3} \\
& \left(\Omega(t)-\Omega_{\text {rated }}\right)^{2} \\
& \left(P_{e l}(t)-P_{\text {rated }}\right)^{2} \\
& \dot{x}_{T}^{2}(t) \\
& \dot{\theta}_{P}^{2}(t) \\
& \dot{\theta}^{2}(t) \\
& \dot{M}_{g}^{2}(t) \\
& +S_{1} \\
& \left(M_{\text {Aero }, y}(t)^{2}+M_{\text {Aero }, z}(t)^{2}\right) \text {. }
\end{aligned}
$$

The first line of (9) penalizes the deviation from the rated rotor speed $\Omega_{\text {rated }}$ and the second line deviation of the electrical power of the generator from its rated value. These are necessary for the control strategy to stabilize the turbine at the desired operating point in full load operation. Line three and four ensure that the wind turbine changes its steady state in a quasi-static manner. The lines multiplied by $R_{i}$ are input costs which penalize $\dot{\theta}, \dot{M}_{g}, \theta_{V}$ and $\theta_{H}$. For weighting the pitch actuator rate $\dot{\theta}$ a scheduled weight is used to account for the higher sensitivity of the pitch at higher wind speed. $R_{2}$ penalizes the deviation of the generator torque and also helps to smooth the generator torque. With $R_{3}$ the vertical and horizontal pitch angles of the IPC approach are penalized to account for the energy cost and the abrasion, which appear when pitching in a cyclic way and $S_{1}$ penalizes the pitching and yawing moment of the rotor.

Furthermore, a set of constraints $H$ guarantees the feasible operation range of the FOWT:

$$
\begin{aligned}
\theta_{\text {min }} \leq \theta(t) & \leq \theta_{\text {max }} \\
-\dot{\theta}_{\text {max }} \leq \dot{\theta}(t) & \leq \dot{\theta}_{\text {max }} \\
M_{g, \text { min }} \leq M_{g}(t) & \leq M_{g, \text { max }}
\end{aligned}
$$

$$
\begin{aligned}
-\theta_{V, \max } \leq \theta_{V}(t) & \leq \theta_{V, \max } \\
-\theta_{H, \max } \leq \theta_{H}(t) & \leq \theta_{H, \max } \\
-M_{y T, \max } \leq M_{y T}(t) & \leq M_{y T, \max } \\
\theta_{V}(t)^{2}+\theta_{H}(t)^{2} & \leq \theta(t)^{2},
\end{aligned}
$$

where (10a) limits the pitch angle to its feasible working area, the second line $(10 \mathrm{~b})$ restricts the pitch rate to $\dot{\theta}_{\max }$, (10c) marks the range in which the generator moment is feasible, the forth and fifth line (10d) and (10e) limit the IPC coordinates $\theta_{V}$ and $\theta_{H}$ to its feasible and admissible range The tower fore-aft bending moment is limited to $M_{y T, \max }$ in (10f) and (10g) guarantees that the commanded pitch angle is always greater than zero by limiting the vertical and horizontal pitch angle $\theta_{V}$ and $\theta_{H}$ with respect to the collective pitch angle $\theta$.

The optimal control problem (8) is converted into a nonlinear program using the Direct Multiple Shooting method, [27]. This can be solved with Sequential Quadratic Programming (SQP). In this work Omuses [28] is used for the large-scale SQP-type nonlinear optimization solver HQP. The prediction time is $T=5 \mathrm{~s}$, which is a realistic preview time of a LIght Detection And Ranging (LIDAR). The sampling time is 0.2 s resulting in $K=25$ discretization stages. These values are chosen heuristically but have already shown promising results in NMPC for FOWT with CPC by [20]. On a PC the mean time for solving the optimization problem is $1.3 \mathrm{~s}$.

\section{SIMULATION RESULTS}

In this section the NMPC is compared to a baseline controller (BC) based on the work of [5] and [2] and to an extended baseline controller with IPC (BIPC) based on the approach of [26]. For both, BC and BIPC the generator torque is held constant above rated wind speed. The control goal is to maintain constant rotor speed at $\Omega_{\text {rated }}=12.1 \mathrm{rpm}$, generator power at $P_{\text {rated }}=5 \mathrm{MW}$ and to reduce structural loads. For the NMPC controller perfect state estimation and a perfect preview on reduced wave and reduced wind disturbances are assumed. State estimation can be realized using observer techniques. LIDAR reconstruction methods can be used to obtain the wind field information effective wind speed and vertical and horizontal linear shear, see [29], however this is out of the scope of this paper. In a first step a nominal simulation scenario where the controlled simulation model is equivalent to the internal model of the NMPC controller is analyzed to evaluate the possibility of minimizing pitching and yawing moments and to reduce rotor speed deviation. Fig. 5 depicts the rotor speed $\Omega$ and pitching $M_{\text {Aero,y }}$ and yawing moment $M_{\text {Aero,z }}$ which are held

TABLE I

OVERVIEW OF CHARACTERISTIC RESULTS OF THE SIMULATIONS AND REDUCTION WITH RESPECT TO THE BC CONTROLLER.

\begin{tabular}{ll|c|c|c||c|c} 
& & BC & BIPC & MPC & BIPC & MPC \\
\hline $\operatorname{DEL}\left(M_{\text {oop }, 1}\right)$ & {$[\mathrm{MNm}]$} & 11.3 & 10.3 & 9.2 & $8.6 \%$ & $18.3 \%$ \\
$\operatorname{DEL}\left(M_{\text {flap }, 1}\right)$ & {$[\mathrm{MNm}]$} & 11.9 & 10.9 & 9.7 & $8.2 \%$ & $18.8 \%$ \\
\hline$\sigma(\Omega)$ & {$[\mathrm{rpm}]$} & 0.83 & 0.86 & 0.15 & $-3.4 \%$ & $81.7 \%$ \\
$\sigma\left(P_{\text {el }}\right)$ & {$[\mathrm{kW}]$} & 343.0 & 354.5 & 76.8 & $-3.3 \%$ & $77.6 \%$
\end{tabular}



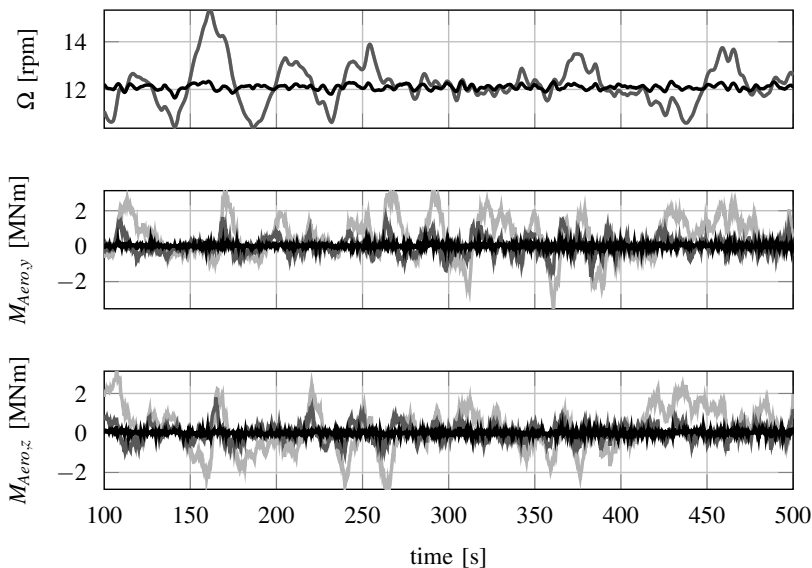

Fig. 5. Different controllers in nominal simulation; Simulation model is the reduced model for individual pitch control. BC (light gray), BIPC (gray), MPC (black).

to zero by the NMPC controller. Small deviations result due to the penalty of vertical and horizontal pitching angle by $R_{3}$.

In a second step, the controllers are compared in a fatigue load simulation with a turbulent wind field with mean wind speed of $16 \mathrm{~m} / \mathrm{s}$ and a turbulence intensity of $15.4 \%$. According to IEC DLC 1.1, irregular waves with significant wave height of $H_{S}=3.37 \mathrm{~m}$ and a peak spectral period of $T_{p}=10.1 \mathrm{~s}$ are applied. All simulations are started in the operation point to avoid transient effects. Fig. 6 and Table I depict that with the chosen set of parameters the NMPC with IPC shows promising results in damping the platform pitch movement and reducing standard deviation of rotor speed $\sigma(\Omega)$ and electrical power $\sigma\left(P_{e l}\right)$ in the same way the NMPC with CPC does, see [20]. Furthermore, it significantly reduces flap-wise blade moments and out-of-plane moments. Fig. 7 shows a PSD analysis of the nonrotating pitching and yawing moments and the flap-wise moment. In $M_{a e r o, y / z}$ peaks at low frequencies representing the asymmetry in the wind field are reduced, see [26]. This yields to a reduction of the frequencies around $0.2 \mathrm{~Hz}(1 \mathrm{P})$ in rotating coordinates.

Considering the DEL (Damage Equivalent Load) of the out-of-plane moment and the flap-wise moment in Table I the reduction shows a promising improvement.

\section{CONCLUSION AND OUTLOOK}

In the presented work an extension of the aerodynamic model for a reduced model of a floating wind turbine with individual pitch control has been developed and implemented in a nonlinear model predictive control framework. The focus is set on the additional reduction of blade loads. The extension is realized as a static nonlinear model describing the pitching and yawing moments. Therefore, influence coefficients are introduced describing the major and the minor coupling. Altogether, the model reproduces the loads in a good manner although several effects of rotating blades are not modeled.
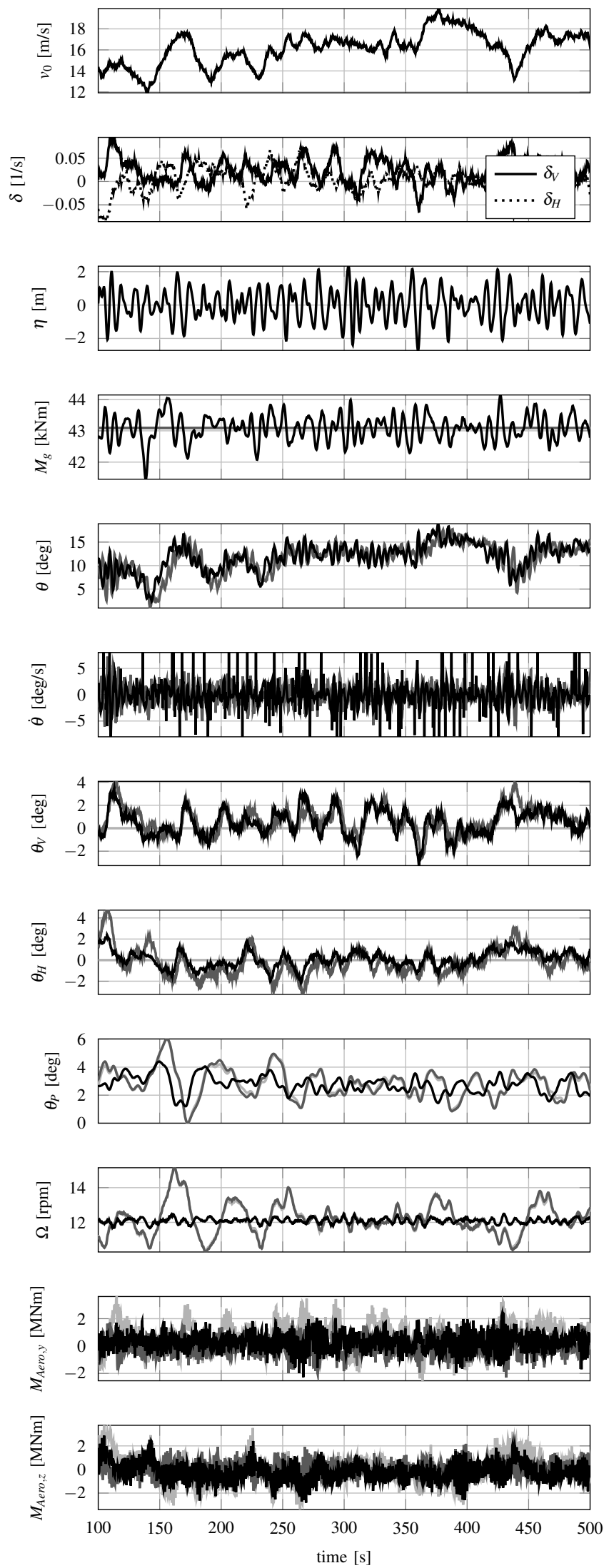

Fig. 6. Simulation results of a fatigue load case simulated with FAST with all DOFs and a turbulent wind. BC (light gray), BIPC (gray), MPC (black). 

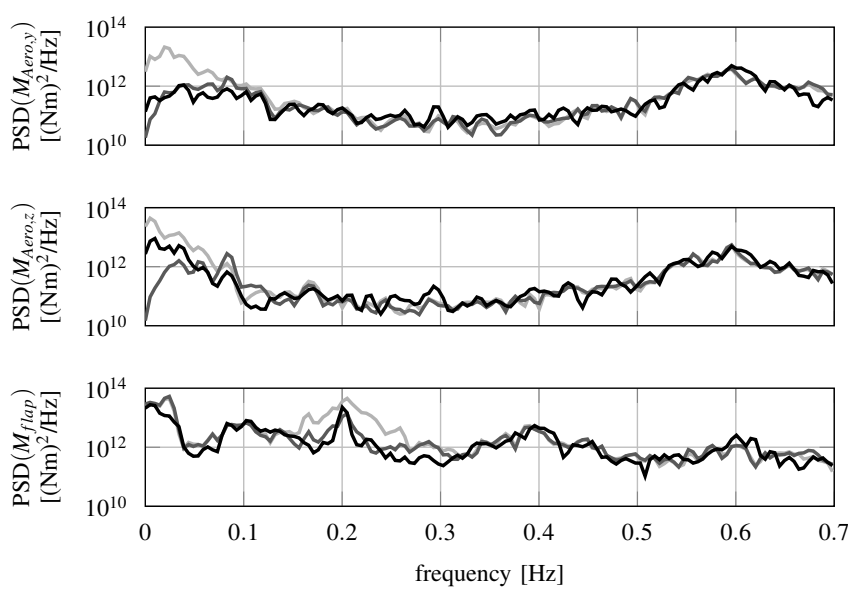

Fig. 7. PSD analysis of pitching moment, yawing moment and the flapwise blade moment in a fatigue load case. BC (light gray), BIPC (gray), MPC (black).

In full load operation optimal input trajectories for the transformed individual pitch angles and the generator torque are calculated assuming perfect state estimation and a perfect preview on reduced wave and wind disturbances. The NMPC controller is then compared to the standard baseline controller and an individual pitch control extension for the baseline controller. The NMPC controller shows promising results reducing significantly the blade loads.

In future work the controller will be tested for a full set of wind fields and waves. Furthermore, robustness considerations should be taken into account as well as state and disturbance estimators.

\section{ACKNOWLEDGMENT}

The presented work was funded by The German Federal Ministry for the Environment, Nature Conservation and Nuclear Safety within the scope of the joint research project LIDAR II.

\section{REFERENCES}

[1] C. Butterfield, W. Musial, and J. Jonkman, "Engineering challenges for floating offshore wind turbines," in the Proceedings of the Copenhagen Offshore Wind Conference, Copenhagen, Denmark, 2005.

[2] J. Jonkman, "Dynamics modeling and loads analysis of an offshore floating wind turbine," National Renewable Energy Laboratory, Golden (Colorado), USA, Tech. Rep. TP-500-41958, 2007.

[3] F. Sandner, D. Schlipf, D. Matha, R. Seifried, and P. W. Cheng, "Reduced nonlinear model of a spar-mounted floating wind turbine," in the Proceedings of the German Wind Energy Conference - DEWEK, Bremen, Germany, 2012.

[4] G. Betti, M. Farina, G. A. Guagliardo, M. Andrea, and R. Scattolini, "Development of a control-oriented model of floating wind turbines," IEEE Transactions on Control Systems Technology, 2013.

[5] T. J. Larsen and T. D. Hanson, "A method to avoid negative damped low frequent tower vibrations for a floating, pitch controlled wind turbine," Journal of Physics: Conference Series, vol. 75, p. 012073, 2007.

[6] J. E. Withee, "Fully coupled dynamic analysis of a floating wind turbine system," Ph.D. dissertation, Massachusetts Institute of Technology, 2004.

[7] E. Wayman, P. Sclavounos, S. Butterfield, J. Jonkman, and W. Musial, "Coupled dynamic modeling of floating wind turbine systems," in Offshore Technology Conference, Houston (Texas), USA, 2006.
[8] E. Lindeberg, "Optimal control of floating offshore wind turbines," Ph.D. dissertation, Norwegian University of Science and Technology, Trondheim, Norway, 2009.

[9] A. Härer, D. Matha, D. Kucher, and F. Sandner, "Optimization of offshore wind turbine components in multi-body simulations for cost and load reduction," in Proceedings of the EWEA Offshore, Frankfurt, 2013, pp. 1-7.

[10] F. Sandner, D. Schlipf, D. Matha, and P. W. Cheng, "Integrated Optimization Of Floating Wind Turbine Systems," in Proceedings of the 33rd International Conference on Ocean, Offshore and Arctic Engineering, 2014.

[11] B. Fischer, "Reducing rotor speed variations of floating wind turbines by compensation of non-minimum phase zeros," in the Proceedings of the European Wind Energy Association Annual Event, Copenhagen, Denmark, 2012.

[12] F. G. Nielsen, B. Skaare, J. O. G. Tande, I. Norheim, and K. Uhlen, "Method for damping tower vibrations in a wind turbine installation," US Patent 20080260514 A1, 2012.

[13] T. Bakka and H. R. Karimi, "Robust output feedback hinf control synthesis with pole placement for offshore wind turbine systems: An lmi approach," in in the Proceedings of IEEE International Conference on Control Applications (CCA), Dubrovnik, Croatia, 2012.

[14] M. Lackner, "An investigation of variable power collective pitch control for load mitigation of floating offshore wind turbines," Wind Energy, vol. 16, no. 3, pp. 435-444, 2013.

[15] H. Namik and K. Stol, "Individual blade pitch control of floating offshore wind turbines," Wind Energy, vol. 13, no. 1, pp. 74-85, 2010.

[16] M. Mirzaei, M. Soltani, N. K. Poulsen, and H. H. Niemann, "An mpc approach to individual pitch control of wind turbines using uncertain lidar measurements," in the Proceedings of the European Control Conference, Zürich, Switzerland, 2013.

[17] L. C. Henriksen, "Model predictive control of wind turbines," Ph.D. dissertation, Technical University of Denmark, Lyngby, Denmark, 2010

[18] D. Schlipf, L. Y. Pao, and P. W. Cheng, "Comparison of feedforward and model predictive control of wind turbines using lidar," in the Proceedings of the 51st Annual Conference on Decision and Control $(C D C)$, IEEE, Maui (Hawai), USA, 2012.

[19] M. D. Spencer, K. A. Stol, C. P. Unsworth, J. E. Cater, and S. E. Norris, "Model predictive control of a wind turbine using shortterm wind field predictions," Wind Energy, vol. 16, pp. 417-434, 2013.

[20] D. Schlipf, F. Sandner, S. Raach, D. Matha, and P. W. Cheng, "Nonlinear model predictive control of floating wind turbines," in the Proceedings of the 23rd International Ocean and Polar Engineering Conference - ISOPE, Anchorage (Alaska), USA, 2013.

[21] J. Jonkman and M. Buhl Jr., "FAST User's Guide," National Renewable Energy Laboratory, Golden (Colorado), USA, Tech. Rep. EL-500$38230,2005$.

[22] W. Schiehlen and P. Eberhard, Technische Dynamik. Wiesbaden, Germany: B.G. Teubner, 2012.

[23] T. Burton, N. Jenkins, D. Sharpe, and E. Bossanyi, Wind Energy Handbook. Chichester, United Kingdom: John Wiley \& Sons, Ltd, 2011.

[24] D. Schlipf, D. J. Schlipf, and M. Kühn, "Nonlinear model predictive control of wind turbines using lidar," Wind Energy, 2012.

[25] P. J. Moriarty and A. C. Hansen, "AeroDyn Theory Manual," National Renewable Energy Laboratory, Golden (Colorado), USA, Tech. Rep. TP-500-36881, 2005.

[26] E. Bossanyi, "Wind turbine control for load reduction," Wind Energy, vol. 6, pp. 229-244, 2003.

[27] R. Findeisen, "Nonlinear Model Predictive Control : A Sampled-Data Feedback Perspective," Ph.D. dissertation, 2004.

[28] R. Franke, "Omuses - a tool for the optimization of multistage systems and hqp a solver for sparse nonlinear optimization," TU Ilmenau, Tech. Rep., 1998.

[29] S. Raach, D. Schlipf, F. Haizmann, and P. W. Cheng, "Three dimensional dynamic model based wind field reconstruction from lidar data," 2014, submitted to The Science of Making Torque from Wind, Kopenhagen, Denmark. 